\title{
Osteoblastos humanos cultivados sobre arcabouços à base de nanotubos de carbono superhidrofílicos e nanohidroxiapatita
}

\author{
Human osteoblasts cultivated on biomineralizated nanohydroxyapatite/superydrophilic \\ carbon nanotube scaffolds
}

Gislene R. Silva ${ }^{1}$, Fernanda Roberta Marciano ${ }^{1}$, Cristina Pacheco-Soares ${ }^{1}$, Anderson de O. Lobo ${ }^{1}$

\begin{abstract}
Resumo
Nanocompósitos à base de nanotubos de carbono verticalmente alinhados de paredes múltiplas superhidrofílicos (VAMWCNT-O) e nano-hidroxiapatita (nHAp) (nHAp/ VAMWCNT-O) são de grande interesse em medicina ósseo regenerativa. A biomineralização utilizando fluido corporal simulado (SBF) vem sendo extensivamente estudado para avaliar a bioatividade de biomateriais. A combinação de nanocompósitos nHAp/VAMWCNT biomineralizados torna-se-ão atraentes e promissores. O objetivo deste estudo foi avaliar a adesão de osteoblastos humanos (HOB) e expressão de fosfatase alcalina in vitro quando cultivados sobre nanocompósitos à base de nHAp/VAMWCNT-O antes e após o período de biomineralização em SBF. A calcificação da matriz extracelular in vitro de células de osteoblastos humanos (HOB) após 7 dias em cultura foi investigada através do ensaio de fosfatase ácida/alcalina. Os arcabouços nHAp/VAMWCNT-O biomineralizados apresentaram maior calcificação da MEC do que os filmes de VAMWCNT-O, nanocompósitos nHAp/VAMWCNT-O e controle de HOB. Estes resultados validam e tornam promissor o processo de biomineralização in vitro dos nanocompósitos nHAp/VAMWCNT-O biomineralizados como arcabouços na regeneração do tecido ósseo.
\end{abstract}

Palavras-chave: Nanotubos de carbono; Nano-hidroxiapatita; SBF; Osteoblasto; Calcificação; Adesão celular.

\begin{abstract}
Nanocomposites based on superhydrophilic vertically aligned multi-walled carbon nanotubes (VAMWCNT-O) and nano-hydroxyapatite ( $\mathrm{nHAp}$ ) are of great interest in bone regenerative medicine. The biomineralization using simulated body fluid (SBF) has been extensively studied to evaluate the bioactivity of biomaterials. The combination of $n H A p$ and VAMWCNT-O is attractive and promising. The aim of this study was to evaluate the in vitro human osteoblast (HOB) adhesion and phosphatase alkaline expression when cultivated on nHAp/VAMWCNT-O nanocomposites before and after the biomineralization into SBF. We also investigated in vitro calcification of the extracellular matrix of $H O B$ cells in culture after 24 hours through the assay of alkaline phosphatase. These promising in vitro results validate biomineralized $n H A p / V A M W C N T-O$ as possible scaffolds for bone tissue regeneration.
\end{abstract}

Keywords: Carbon nanotubes; Nano-hydroxyapatite; SBF; Osteoblast; Calcification; Cell adhesion. 


\section{Introdução}

Nanotubos de carbonos (CNT) são estruturas tubulares formadas por arranjos hexagonais de carbono, podendo ser classificados em dois tipos: nanotubos de carbono de paredes simples (SWCNT) e nanotubos de carbono de paredes múltiplas (MWCNT) ${ }^{(1-6)}$. Os MWCNT são de particular interesse na medicina regenerativa por preservar as propriedades biológicas e permitirem o extenso crescimento, espalhamento e adesão de células, além de possuirem excelente biocompatibilidade celular ${ }^{(7)}$. Estudos com MWCNT demonstram que os materias contem caracteristica e propriedades que suportam o crescimento de células osteoblásticas, estimulando a produção de matriz extracelular (MEC) na formação de tecido ósseo e o crescimento de cristais de hidroxiapatita ${ }^{(8)}$.

As propriedades de biocompatibilidade dos MWCNT ficam melhores quando se esta na forma alinhada (VAMWCNT) e com superhidrofilicidade $(\text { VAMWCNT-O) })^{(4)}$.

Os VAMWCNT-O têm uma combinação excepcional de propriedades mecânicas e químicas, elevada relação de aspecto, a porosidade nanométrica e biocompatibilidade $^{(5)}$. Esta combinação e seu tamanho nanométrico tem o papel importante no estudo da regeneração de tecido ósseo.

A hidrofilicidade é um fator que favorece diretamente os eventos celulares intrinsicos. A melhor molhabilidade aumenta a superficie de contato entre os nanobiomateriais e as proteínas de adesão celular. Esses fenômenos são importantes para que ocorra a migração, proliferação e diferenciação celular ${ }^{(4)}$.

A nano-hidroxiapatita (nHAp) é um mineral que favorece o crescimento osseo, é biocompatível e bioativo, e tem aplicação na regeneração óssea por estabelecer ligações químicas entre o material e o tecido osseo permitindo a proliferação celular ${ }^{(4)}$. A associação de VAMWCNT-O com a nHAp tornam-se emergentes para associar o potencial biomimético com propriedades mecânicas ${ }^{(5)}$.

Além da aplicação dos nanocompósitos nHAp/ VAMWCNT-O como arcabouços, o processo de biomineralização de biomateriais realizado pela incubação das amostras em SBF (com concentrações iônicas semelhantes ao líquido extracelular humano) auxilia no desenvolvimento de apatitas biológicas na superfície dos nanocompósitos nHAp/VAMWCNT-O melhorando a adesão e proliferação celular em ensaios in vitro com células de osteoblastos humanos ${ }^{(9-12)}$.

Os osteoblasto são células de revestimentos responsáveis pela síntese dos componentes orgânicos da matriz óssea. Liberam fibronectinas que têm como característica a habilidade de formar a calcificação óssea e aumentar a liberação de fosfatase alcalina ${ }^{(13)}$.

A fosfatase alcalina é uma enzima responsável pela proliferação e renovação celular, esta distribuída nos tecidos humanos, principalmente na mucosa intestinal, fígado (canalículos biliares), túbulos renais, baço, ossos (osteoblastos) e placenta, de modo que nos ossos a atividade da mesma está confinada aos osteoblastos onde ocorre a formação óssea ${ }^{(14)}$.

\section{Objetivo}

O objetivo deste trabalho foi avaliar o potencial de calcificação da MEC de osteoblastos humanos cultivados sobre a nova classificação de nanobiomateriais representada pelos nanocompósitos de nHAp/VAMWCNT-O biomineralizados visando sua utilização como arcabouços na regeneração do tecido ósseo.

\section{Metodologia}

O processo de produção dos VAMWCNT foi realizado no Laboratório Associado de Sensores e Materiais do Instituto Nacional de Pesquisas Espaciais. Para o crescimento do filme de VAMWCNT utilizou-se um reator de plasma de microondas. Utilizaram-se ligas de TiAl4V (ASTM, F136) como substrato. Como catalisadores metálicos, para consequente nucleação dos VAMWCNT, utilizou-se o depósito de uma camada de $10 \mathrm{~nm}$ de Ni Os VAMWCNT foram nucleados em duas etapas, denominadas, pré-tratamento e deposição, respectivamente. $\mathrm{Na}$ etapa de pré-tratamento, o objetivo foi criar nanoilhas do material catalisador, a partir das quais os nanotubos puderam ser nucleados. Antes de iniciar esse processo, foram inseridos $90 \mathrm{sccm}$ (centímetros cúbicos por minuto) de $\mathrm{H}_{2}+10 \mathrm{sccm}$ de $\mathrm{N}_{2}$ a uma pressão de $4 \times 10^{3} \mathrm{~Pa}$, aquecendo o porta-amostra com uma resistência elétrica até atingir uma temperatura aproximada de $300^{\circ} \mathrm{C}$. Na fase de pré-tratamento, com duração de 5 minutos, a potência do gerador de microondas foi mantida em $800 \mathrm{~W}$ e a 
temperatura atingiu cerca de $760^{\circ} \mathrm{C}$. Na etapa de deposição, $14 \mathrm{sccm}$ de $\mathrm{CH}_{4}$ foram adicionados como fonte de carbono por 1 minuto $^{(4)}$.

Para obtenção da superhidrofilicidade, ocorreu a funcionalização dos VAMWCNT (VAMWCNT-O), utilizando o sistema de plasma de $\mathrm{O}_{2}$ DC-pulsado, nos parâmetros experimentais desenvolvido por $\mathrm{Lobo}^{(4)}$ : Tempo de tratamento a plasma: 2 minutos, Tensão aplicada: - $400 \mathrm{~V}$ e Pressão de trabalho: 80 mTorr.

Utilizou-se a técnica de eletrodeposição de nHAp sobre os VAMWCNT-O2, desenvolvidas por Lobo ${ }^{(4)}$. A eletrodeposição de nHAp foi realizada no Laboratório de Nanotecnologia Biomédica do Instituto de Pesquisa e Desenvolvimento (IP\&D) da Universidade do Vale do Paraíba (UNIVAP). Para a produção do eletrólito utilizou-se os reagentes $\mathrm{Ca}\left(\mathrm{NO}_{3}\right)_{2} \cdot 4 \mathrm{H}_{2} \mathrm{O}$ e $\mathrm{NH}_{4} \mathrm{H}_{2} \mathrm{PO}_{4}$ $\left(\right.$ Sigma-Aldrich $^{\circledR}$ ). Salienta-se que para a preparação do eletrólito utilizou-se a razão $\mathrm{Ca} / \mathrm{P}$ da nHAp estequiométrica. $\mathrm{O}$ pH da solução eletrolítica foi em torno de 4,8 .

O processo de deposição eletroquímica foi realizado em um Potenciostato/Galvanostato (AUTOLAB, PGSTAT $128 \mathrm{~N}$, HOLANDA), operando em modo Potenciostático.

Para a eletrodeposição de nHAp, foram utilizado como ânodo VAMWCNT-O (15mm x $15 \mathrm{~mm}$ x $1 \mathrm{~mm})$ acoplado à um porta-eletrodo (formato de cachimbo), de teflon, com uma área exposta, um círculo com $2,8 \times 10^{-5} \mathrm{~m}^{2}$. O processo foi realizado com uma estação de trabalho eletroquímica fornecendo uma energia de corrente contínua no potencial de $-2,0 \mathrm{~V}$, durante 1800 segundos. Utilizou-se como contra-eletrodo um bastão de platina com $5 \times 10^{-2} \mathrm{~m}$ de comprimento e $2 \times 10^{-4} \mathrm{~m}$ de diâmetro. Manteve-se a temperatura do eletrólito à $80^{\circ} \mathrm{C}$ durante todo o processo.

A biomineralização dos nHAp/VAMWCNT-O foi realizada pela imersão das amostras em solução de SBF. A solução foi preparada pela mistura de íons em água destilada, em chapa de agitação, medindo o pH com o auxílio do peagâmetro, para obtenção do $\mathrm{pH}$ 6,12 em temperatura de $28^{\circ} \mathrm{C}$. A composição do $\mathrm{SBF}$ está apresentada na Tabela 1.

Tabela 1. Quantidade de reagentes para a preparação do SBF.

\begin{tabular}{c|c} 
Reagente & Quantidade (mM) \\
\hline $\mathrm{NaCl}$ & 733,5 \\
\hline $\mathrm{MgCl}_{2}$ & 7,5 \\
\hline $\mathrm{CaCl}_{2} \cdot 2 \mathrm{H}_{2} \mathrm{O}$ & 12,5 \\
\hline $\mathrm{Na}_{2} \mathrm{HPO}_{4} \cdot 2 \mathrm{H}_{2} \mathrm{O}$ & 5 \\
\hline $\mathrm{NaHCO}_{3}$ & 21 \\
\hline
\end{tabular}

Os nanocompósitos de nHAp/VAMWCNT-O alocados nos respectivos tubos corning foram levados à câmara de fluxo laminar de radiação ultravioleta (UV) (modelo BioProtector-12 Plus VECO) por 30 minutos. Após este período, com o auxílio de uma pinça, cada amostras foi colocada em um tubo corning, com $20 \mathrm{~mL}$ de SBF, e acondicionadas em incubadora refrigerada de bancada (marca Cientec, modelo CT-712-R). Os tubos foram agitados na incubadora à $75 \mathrm{rpm}$, em temperatura de $36,5^{\circ} \mathrm{C}$ pelo periodo de 7, 14 e 21 dias, para ocorrer o processo de biomineralização dos nHAp/VAMWCNT-O. Após a incubação as amostras foram retiradas da solução de $\mathrm{SBF}$ e levadas a estufa (modelo sp400 SPLABOR) a $50^{\circ} \mathrm{C}$ para secagem.

Para os ensaios biológico foram utilizados células de HOB cultivadas, em garrafas de cultura de celulas de $25 \mathrm{~cm}^{3}$ em Meio DMEM baixa glicose, suplementado com $10 \%$ de soro fetal bovino e $1 \%$ de antibiotico antimicótico, incubados em estufa (modelo 3110 Forma Scientific), em atmosfera controlada de $\mathrm{CO}_{2}$ a $5 \%$ e temperatura a $37^{\circ} \mathrm{C}$ para obter a população ideal para o experimento. As células de HOB foram replicadas a partir da contagem inicial e o meio de cultura substituído a cada 48 horas, para melhores condições de proliferação e viabilidade celular. Os testes de cultura de células foram desenvolvidos no Laboratório de Dinâmica de Comportamento Celulares do IP\&D da UNIVAP, supervisionados pela Prof ${ }^{\text {a }}$ Dra. Cristina Pacheco-Soares.

Sobre As células HOB foi adcionado tripsina por 3 minutos, feito jatos para soltar as células da garrafa de cultura, com auxilio de uma pipeta foi colocada em tubos corning e centrifugadas por 5 minutos para separação das células, em seguida foram resuspendidas em Meio DMEM baixa glicose (Gibco), suplementado com 10\% de soro fetal bovino e $1 \%$ de antibiótico antimicótico. O volume de $10 \mu \mathrm{L}$ foi aplicado à Câmara de Newbauer e levado a contagem das células de HOB no microscópio invertido (marca Olympus CK 40) e determinada a concentração de 4,0 x $105 \mathrm{cel} / \mathrm{mL}$. As amostras foram dividas em 4 grupos: VAMWCNT-O, nHAp/VAMWCNT-O e nHAp/VAMWCNT-O biomineralizados nos respectivos tempos de incubação (7, 14 e 21 dias) e o controle (células de HOB). A seguir, as amostras foram distribuidas nos poços de 3 placas de 24 poços e semeadas sobre cada uma a concentração de 4,0 x $105 \mathrm{cel} / \mathrm{mL}$ e levadas a estufa a $37^{\circ} \mathrm{C}$ com $5 \%$ de $\mathrm{CO}_{2}$ por 30 minutos para proporcionar maior adesão. Após o periodo de adesão, nas amostras 
foram acrescentadas meio DMEM baixa glicose (Gibco) até completar o volume de $500 \mu \mathrm{L}$ e incubadas na estufa nos tempos de 24 horas, 48 horas e 7 dias. Todos os testes foram realizados em triplicata.

Para o teste de atividade da Fosfatase alcalina (ALP), foram preparadas as soluções 1 e 2 do Kit de ensaio de Fosfatase Ácida/Alcalina (marca Millipore), conforme o manual do produto. Nos tempos determinados ( 24 horas, 48 horas e 7 dias) as placas foram retiradas da estufa, em cada poço foram adicionados $25 \mu \mathrm{L}$ da solução 2 e $100 \mu \mathrm{L}$ da solução 1 , a placa foi coberta com papel alumínio e aguardou 15 minutos em tempertatura ambiente. A seguir as amostras foram levadas para análise imunohistoquímica em aparelho Leitor de microplacas (Synergy HT Multi-Detection, marca BioTek, Winooski, VT) na absorbância de 620nm.

Para a realização das análises estatísticas foram coletadas quatro diferentes leituras, com $\mathrm{N}=4 \mathrm{e}$, expressos como a média. Para a quantificação da calcificação da matriz extracelular utilizou-se o teste de ALP. As diferenças estatísticas foram analisadas pelo Programa Origin $8.0^{\circledR}$. A população dos grupos foi analisada com distribuição normal e independente para cada experimento. Os valores de $\mathrm{p}$ menores que 0,005 foram considerados para indicação de diferenças estatísticas dos grupos analisados.

Para os testes de adesão celular sobre o nanocompósito nHAp/VAMWCNT-O as células HOB cultivadas e semeadas em cada poço a $5 \times 105$ células / $\mathrm{mL}$, suplementado com $10 \%$ de PBS. A incubação foi realizada sob $\mathrm{CO}_{2}$ atmosfera $(5 \%)$ a $37^{\circ} \mathrm{C}$.

Para a análise da adesão por Microscopia de Varredura as células foram fixadas com 3\% de glutaraldeido $0.1 \% \mathrm{M}$ cacodilato de tampão, durante 1 hora e desidratado em série graduada de solução em etanol $(30 \%, 50 \%, 70 \%, 95 \%$ e $100 \%)$ durante 10 minutos. A fase de secagem utilizada uma solução de 1:1 de etanol com hexametildisilazano (HMDS) e por último o HMDS puro e deixado evaporar a temperatura ambiente, depois foi realizado uma fina camada de ouro, para serem examinadas no MEV (modelo JEOL-JSM 5610 VPI). As micrografias foram coletadas com ampliação de $1.000-4.000 \mathrm{x}$.

Para análise imunohistoquimica as células foram fixadas com soluções contendo: $1,1 \mathrm{~mL}$ de tampão cacodilato de sódio, $100 \mathrm{~mL}$ de glutaraldeído e $800 \mathrm{~mL}$ de paraformaldeído a $4 \% \mathrm{~mL}$. Para a imunomarcação as amostras foram incubadas com anticorpo primário Faloidina (Sigma-Aldrich). incubado a temperatura ambiente, no escuro, por 1 hora. Após este período as amostras foram lavadas com PBS em temperatura ambiente. Para a marcação do DNA foi utilzado o DAPI (4'6-diamidino-2-phenylindole, Sigma-Aldrich) por 10min. Após, o microscópio confocal invertido de fluorescência (LSM 510 Axiovert $200 \mathrm{M}$, Carl Zeiss) foi utilizado nos comprimentos de onda: $\lambda$ de $560 \mathrm{~nm}$ (vermelho) e $\lambda$ de $460 \mathrm{~nm}$ (azul), de acordo com o fluorocromo.

\section{Resultados}

Os ensaios de liberação e atividade da ALP foram realizados com o objetivo de avaliar o potencial de calcificação da MEC. A ALP é avaliada pela liberação da detimolftaleína por hidrólise do substrato de timolftaleína monofosfato.

A Fig. 1A apresenta os resultados da atividade de ALP nas amostras: VAMWCNT-O, nHAp/VAMWCNT e nHAp/VAMWCNT biomineralizados nos respectivos tempos de incubação (7, 14 e 21 dias) e o controle. Os grupos de amostras foram utilizados para comparações de calcificação da matriz, buscando a validação dos nanobiomateriais desenvolvidos, seja pela aplicação de nHAp e/ou pelo processo de biomineralização aplicado. As células de HOB incubaram no material por 24 horas, 48 horas e 7 dias, em todos os grupos analisados.

A Fig. 1B apresenta os resultados da calcificação da MEC em porcentagem em relação ao controle (células) e expressos em porcentagem de acordo com cada grupo apresentadas em ( $\mu \mathrm{mol}$ de timoftaleina $/ \mathrm{h} / \mathrm{mg})$.

O teste de adesão foi realizado com o objetivo de mostrar a interação das células cultivadas sobre os nanobiomateriais.

AFig. 2 apresenta as micrografias da HOB cultivadas sobre os nHAp/VAMWCNT-O biomineralizados nos tempos de 7, 14 e 21 dias na Microscopia de Varredura. A micrografia 2A apresenta a célula com suas projeções citoplasmáticas, mostrando um bom processo de proliferação, também é possível observar cristais de nHAp sobre nHAp/VAMWCNT-O-7D. Está muito evidente na micrografia $2 \mathrm{~B}$ a interação da célula com os cristais de nHAp, onde é possível observar todas projeções citoplasmáticas entre os cristais do nHAp/VAMWCNTO-14D. A Fig. 2C apresenta a HOB com suas projeções alongadas fazendo interação com os cristais de nHAp.

A Microscopia de Fluorescência foi apresentada na Fig. 3. Na Fig. 3A é possível ver em azul o núcleo celular bem evidente. A Fig. 3B apresenta o citoplasma celular em vermelho. 


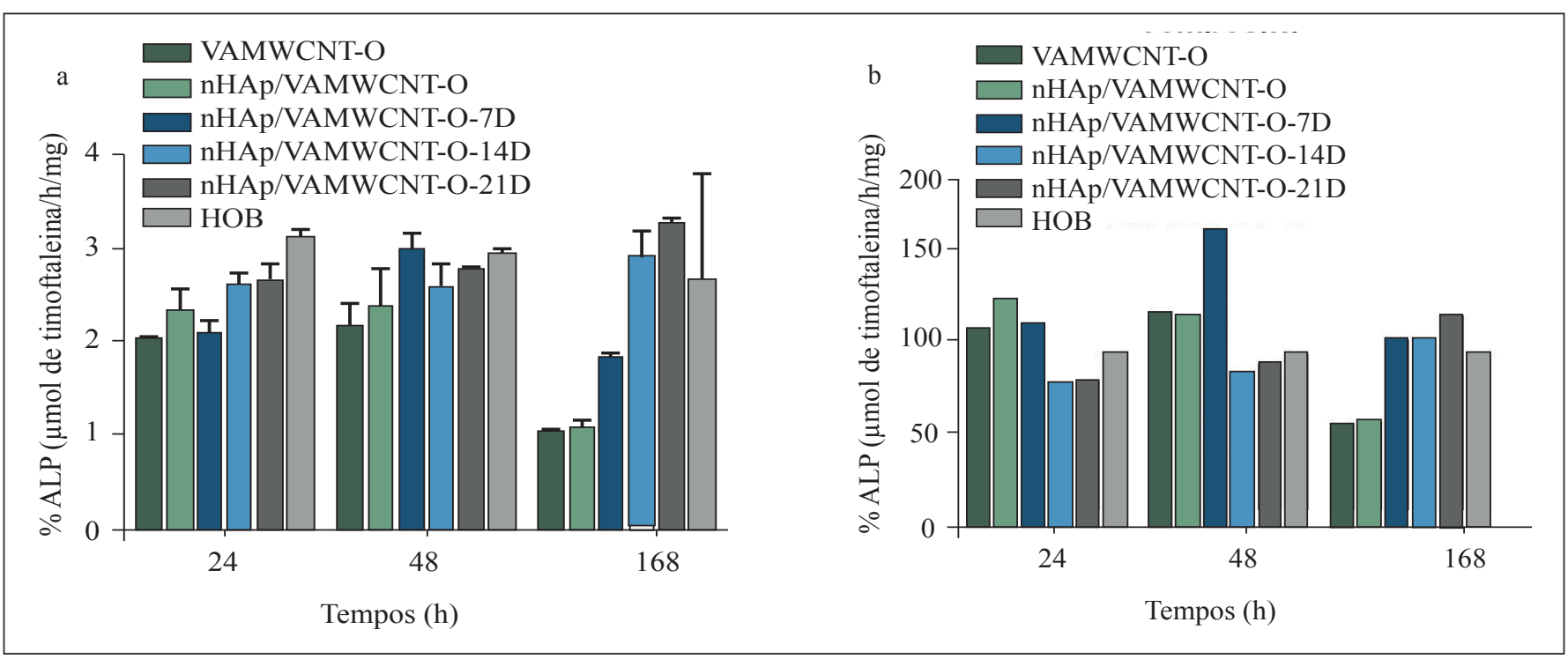

Figura 1. Comparação do crescimento celular nas amostras em absorbância de 620nm (a) e Comparação da calcificação da MEC em porcentagem (a).
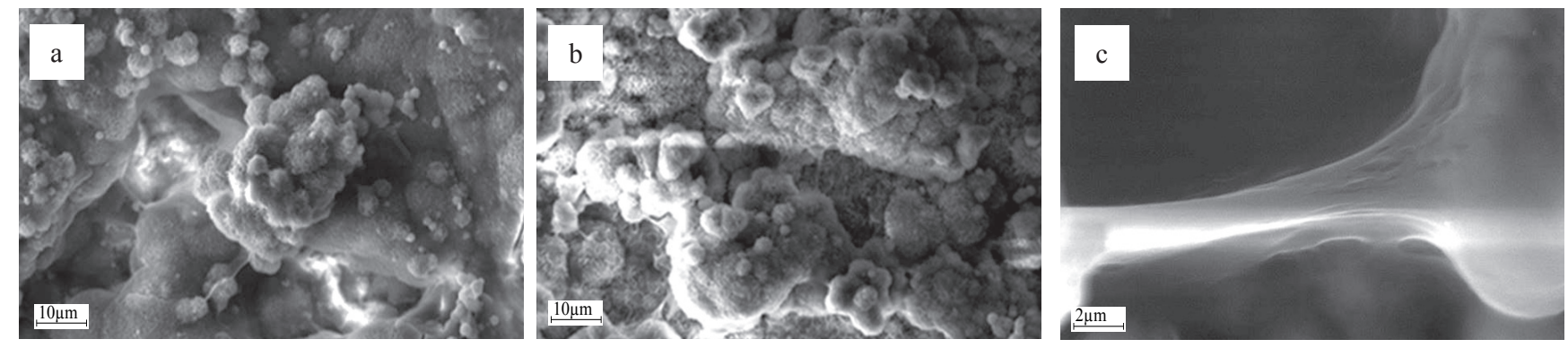

Figura 2. Micrografias da HOB sobre o nHAp/VAMWCNT-O biomineralizados, (a) 7 dias, (b) 14 dias e (c) 21 dias.
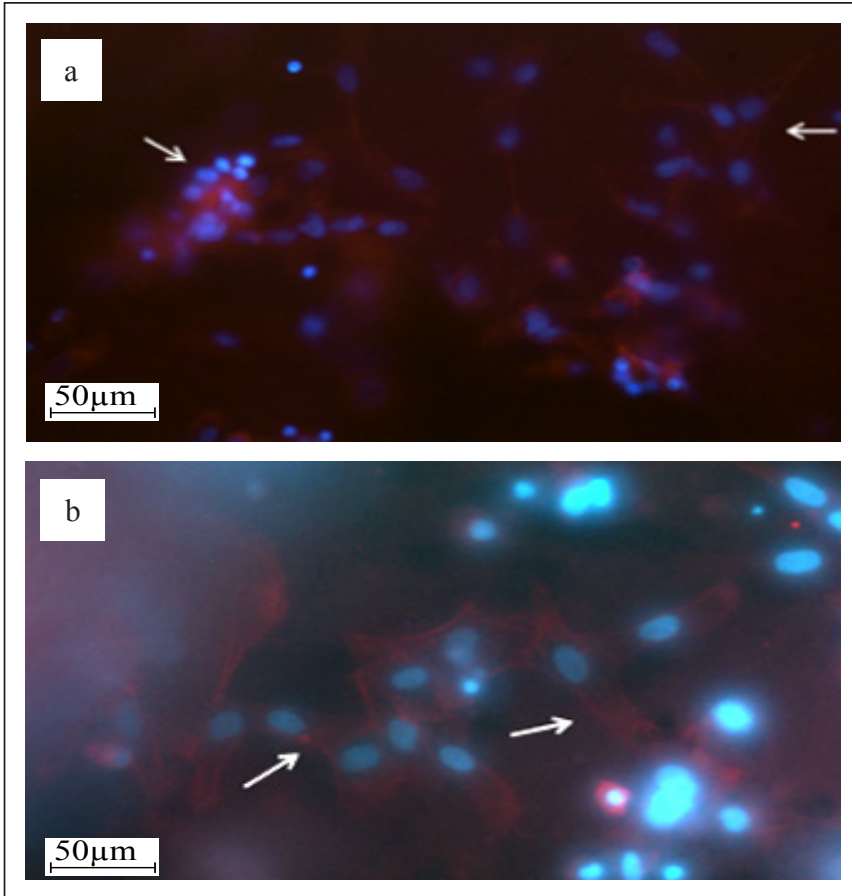

Figura 3. Microscopia de Fluorescência, (a) Núcleo da célula em azul e (b) Citoplasma celular em vermelho.

\section{Discussão}

Na comparação entre os grupos, identificou-se que a ALP apresentou valores superiores para nHAp/VAMWCNT biomineralizados por 14 e 21 dias de incubação em SBF, identificadas a partir de $48 \mathrm{~h}$ de incubação com as células de HOB. Verifica-se no período de 7 dias de incubação com HOB uma elevada calcificação da MEC para as amostras de nHAp/VAMWCNT biomineralizados por 14 e 21 dias de incubação em SBF.

A amostra de nHAp/VAMWCNT biomineralizado por 7 dias de SBF apresentou um pico de calcificação da MEC com 48h de incubação de HOB, porém declinou no período de 7 dias de incubação com HOB. As demais amostras não apresentaram potencial de calcificação da MEC tão intenso quanto as amostras de nHAp/VAMWCNT biomineralizados por 7, 14 e 21 dias de incubação em $\mathrm{SBF}^{(11)}$. 
Xiaoming e colaboradores ${ }^{(15)}$ realizaram o teste da ALP em CNT e observaram no seu estudo que as celulas de osteoblasto apresentaram maior proliferação nas amostras de VAMWCNT do em outro biomaterial como foi possivel visualizar no nosso trabalho afirmando o que ja foi encontrado na literatura.

Em outros estudos ${ }^{(15,16)}$. para a analise de ALP utilizaram uma absorbância de $405 \mathrm{~nm}$, em nosso estudo realizamos análises da ALP em 3 absorbâncias diferentes e tivemos um melhor resultado com a absobância de $620 \mathrm{~nm}$ conforme indicação do produto.

A Fig. 1B mostra os valores na comparação da calcificação da MEC em porcentagem das amostras estudandas. Como mostra que as amostras de nHAp/VAMWCNT biomineralizados apresentaram maior expressão de ALP na cultura de células osteoblásticas humanas.

Nos tempos de 24 e 48 horas os VAMWCNT, nHAp/VAMWCNT e nHAp/VAMWCNT biomineralizados de 7 dias apresentaram uma calcificação da MEC acima de $13 \%$ do controle (celulas), as amostras de nHAp/VAMWCNT biomineralizados de 14 e 21 dias apresentaram 17\% menores que as celulas.

Nos tempo de 7 dias as amostras nHAp/VAMWCNT biomineralizados de 7, 14 e 21 dias apresentaram 7\% a mais que as celulas na calcificação MEC, sendo que no nHAp/VAMWCNT biomineralizados de 21 dias de incubção de SBF apresentou $22 \%$ da calcificação da MEC superior as celulas. Já as amostras VAMWCNT e nHAp/VAMWCNT teve uma queda de $41 \%$ na calcificação tendo as celulas como controle.

\section{Referências}

1. Barbosa, G.M., Avaliação dos efeitos de nanotubos de carbono de parede múltipla carboxilados em células do sistema imunológico-Santa Maria, 2010.50f.: il. Dissertação(Mestrado em Nanociências) - Centro Universitário Franciscano, 2010.

2. Filho, A. G. S.; Fagan, S.B., Funcionalização de nanotubos de carbono, Quim. Nova, Vol. 30, No. 7, 1695-1703, 2007

3. Herbst, M. H.; Macêdo, M. I. F.; Rocco, A. M., Tecnologia dos nanotubos de carbono: tendências e perspectivas de uma área multidisciplinar, Quim. Nova 2004, 27, 986.

4. Lobo, A.O., Obtenção de super-hidrofilicidade em nanotubos de carbono alinhados e sua aplicação como nanobiomaterial - São José dos - Campos, 2011. 208f. Tese de doutorado - Instituto Tecnológico de Aeronáutica, 2011.
Como foi possivel ver, as amostras de nHAp/VAMWCNT biomineralizados de 7 dias apresentaram melhor calcificação da matriz celular em todos os tempos.

Os testes de ALP, MEV e MF mostraram que as propriedades fisico-quimicas do VAMWCNT e nHAp favorecem na proliferação e adesão celular ${ }^{(17)}$.

\section{Conclusão}

Estes resultados indicam que os nanocompósitos de nHAp/VAMWCNT biomineralizados apresentaram maior expressão de ALP na cultura de células osteoblásticas humanas (HOB), indicando potencial de biomineralização in vitro diferenciado em relação ao controle de células e as amostras de VAMWCNTs não biomineralizados. A expressão de ALP foi mais evidente e crescente nas amostras que estiveram em solução de SBF por 14 e 21 dias, de forma que neste período de incubação se obteve um referencial expressivo sobre a calcificação da MEC induzida pelo processo de biomineralização em solução de SBF.

\section{Agradecimentos}

Os autores agradecem a Fundação de Amparo à Pesquisa do Estado de São Paulo (FAPESP) (2011/17877-8) e (2011/20345-7) pelo suporte financeiro e ao Laboratório Associado de Sensores e Materiais do Instituto Nacional de Pesquisas Espaciais pelo espaço cedido para a produção e modificação superficial dos nanotubos de carbono.

5. Qia H. J. et al., Determination of mechanical properties of carbon nanotubes and vertically aligned carbon nanotube forests using nanoindentation. J Mech Phys Solids. 2003;51(11-12):2213-37

6. Pereira, P.C.D., Biocompatibilidade e principais aplicações dos nanotubos de carbono - Belo Horizonte, 2009. Monografia do título de Especialista em Microbiologia Universidade Federal de Minas Gerais, 2009.

7. Oliveira,V. et al., Nanotubos de carbono aplicados as neurociencias: perspectivas e desafios. Rev Psiq Clín. 2011;38(5):201-6

8. Beuvelot, J. et al., In vitro calcification of chemically functionalized carbon nanotube. Acta Biomaterialia 6 (2010) 4110-4117 
9. Mendes, R. M. et al., Effects of single wall carbon nanotubes and its functionalization with sodium hyaluronate on bone repair. Life Sciences 87 (2010) 215-222

10. WEeI, G. et al., Biomimetic growth of hydroxyapatite on super water-soluble carbon nanotube-protein hybrid nanofibers. Carbon 49 (2011) 2216-2226

11. Irineu, J.A.F. et al., Efficient method to produce biomineralizated nanohy- droxyapatite/vertically aligned multiwalled carbon nanotube scaffolds. Materials Letters 79 (2012) 166-169

12. Marsi, T.C.O., Biomineralização de nanotubos de carbono superhidrofílicos verticalmente alinhados - São José dos Campos,2012.Dissertação de Mestrado em Engenharia Biomédica - Universidade do Vale do Paraíba, 2012.

13. Rosa, Z.M.A., Isolamento, cultivo, caracterização citoquimica e resposta de células osteoblásticas à irradiação de Laser de Baixa Potência - São José dos Campos, 2004.106p.:Il.
Dissertação de Mestrado em Engenharia Biomédica Universidade do Vale do Paraíba, 2004.

14. Motta,V.T., Bioquímica Clínica: Princípios e Interpretações - Disponivel em http://www.labclinisul.com.br/artigos/ Bioq.Clinica\%20-\%20Enzimas.pdf acesso em 29/08/12.

15. Xiaoming, L. et al., The use of carbon nanotubes to induce osteogenic differentiation of human adiposederived MSCs in vitro and ectopic bone formation in vivo. Biomaterials 33 (2012) 4818 e 4827.

16. Yan, L. et al., Resveratrol-conjugated poly-e-caprolactone facilitates in vitro ineralizationand in vivo bone regeneration. Acta Biomaterialia 7 (2011) 751-758.

17. Lobo, A.O. et al., An evaluation of cell proliferation and adhesion on vertically-aligned multi-walled carbon nanotube films. Carbon48, (2010) $245-254$. 